А. А. Саченко ${ }^{1,5}$, В. В. Кочан ${ }^{1}$, В. С. Харченко ${ }^{2}$, М. А. Ястребенецкий ${ }^{3}$, Г. В. Фесенко ${ }^{4}$, М. Э. Яновский ${ }^{2}$

${ }^{1}$ Тернопольский национальный экономический университет, г. Тернополь, Украина

${ }^{2}$ Национальный аэрокосмический университет “ХАИ» имени Н. Е. Жуковского, г. Харьков, Украина

${ }^{3}$ Государственный научно-технический центр по ядерной и радиационной безопасности, г. Киев, Украина

${ }^{4}$ Харьковский национальный университет городского хозяйства имени А. Н. Бекетова, г. Харьков, Украина

5 Технический университет Силезии, Гливице, Польша

\section{Система послеаварийного} мониторинга АЭС с использованием беспилотных летательных аппаратов: концепция, принципы построения

Предложена концепция проектирования системы послеаварийного мониторинга оборудования и территории АЭС после тяжелой аварии с использованием беспилотных летательных аппаратов (БПЛА). Проводная сеть определена как наиболее уязвимая в ходе мониторинга аварий. В качестве основных характеристик системы рассматри ваются информативность, живучесть и достоверность. Описываются меры по их обеспечению.

Ключевые слова: АЭС, беспилотный летательный аппарат (БПЛА), послеаварийный мониторинг, информативность, надежность, достоверность, дроны.

А. О. Саченко, В. В. Кочан, В. С. Харченко, М. О. Ястребенецький, Г. В. Фесенко, М. Е. Яновський

Система післяаварійного моніторингу атомних електростанцій з використанням безпілотних літальних апаратів: концепція, принципи побудови

Запропоновано концепцію проектування системи післяаварійного моніторингу обладнання та території АЕС після тяжкої аварії з викорис танням безпілотних літальних апаратів (БПЛА). Провідна мережа визначена як найбільш вразлива в ході моніторингу аварій. В якості основних характеристик системи розглядаються інформативність, надійність і достовірність. Описуються заходи щодо їх забезпечення.

Ключові слова: AEC, безпілотний літальний апарат (БПЛА), післяаварійний моніторинг, інформативність, надійність, достовірність, дрони.

(C) А. А. Саченко, В. В. Кочан, В. С. Харченко, М. А. Ястребенецкий, Г.В.Фесенко, М. Э. Яновский, 2017
Д ля получения оперативной и достоверной информации в критических инфраструктурах (КИ) в последнее время все более активно используются беспилотные летательные аппараты (БПЛА). С их помощью осуществляются [1-8]: мониторинг состояния транспортных магистралей, нефте- и газопроводов, линий электропередачи и других объектов КИ;

инженерная разведка районов наводнений, землетрясений и других стихийных бедствий на объектах КИ;

определение точных координат пострадавших объектов КИ;

доставка малогабаритных специальных грузов и средств, необходимых для проведения аварийно-спасательных работ и жизнеобеспечения персонала объектов КИ при чрезвычайных ситуациях и террористических актах на них;

выяснение степени химического (на химических опасных объектах) или радиоактивного (на радиационных опасных объектах) заражения местности с установлением точных данных о концентрациях вредных веществ и уровнях опасных излучений для определения возможности направления туда спасателей, для выбора времени и режима их работы, а также средств индивидуальной защиты;

патрулирование территории КИ с целью предотвращения несанкционированного доступа на эту территорию.

Преимущества использования БПЛА в перечисленных случаях:

высокая оперативность, что особенно важно в аварийной ситуации;

исключение или существенное снижение угрозы для жизни и здоровья персонала;

визуальный осмотр объектов в режиме реального времени и обеспечение высокого качества изображений в сложных послеаварийных условиях;

возможность измерения эмиссии и выбросов опасных веществ в труднодоступных зонах объектов;

использование термографии и ночного видения;

экономически эффективное решение благодаря относительно невысокой стоимости БПЛА;

возможность группового применения БПЛА для реализации различных функций в условиях изменения послеаварийной ситуации.

БПЛА все шире применяются и в атомной энергетике для повышения эффективности мониторинга АЭС как до аварии, так и после ее возникновения. Например, японские специалисты предложили систему мониторинга АЭС [3], которая включает в себя БПЛА (самолеты, вертолеты, дроны), снабженные видеокамерами и приборами радиационной разведки и дозиметрического контроля, радиозонд, пилотируемый вертолет и другие устройства. БПЛА, для определения качества дезактивационных работ на этапе ликвидации последствий аварии, программируются на периодический контроль радиационной обстановки над одними и теми же объектами АЭС, имея функцию зависания над ними и возможность передавать собранные данные в реальном масштабе времени в кризисный центр.

Американские ученые разработали прототип БПЛА для проведения радиационной разведки с целью идентификации мест наибольшего излучения и картирования обстановки при аварии на АЭС [4]. Оснащенный специальными микроконтроллерами, БПЛА по специальным протоколам передает информацию в кризисный центр, где она обрабатывается и используется для принятия решений по управлению ликвидацией аварии.

Заслуживает внимания используемый для решения задач мониторинга АЭС российский многоцелевой 
беспилотный самолет Supercam S350 [5] с продолжительностью полета до 4 ч, имеющий навигационные огни, встроенную цифровую систему телеметрии, радиомодем с приемником, систему самодиагностики, систему автоматического отцепа крыльев. Данный беспилотный самолет, разворачиваясь за 10 мин, может выполнять задачи на рабочей высоте 50-500 м и на расстоянии до 350 км в условиях ветра со скоростью до 15 м/с, умеренного дождя и снегопада.

Вместе с тем, применение БПЛА порождает целый ряд проблем, связанных с необходимостью:

разработки национальных и международных стандартов с требованиями к надежности и безопасности использования БПЛА;

комплектования БПЛА техническими средствами обнаружения воздушных объектов с целью недопущения столкновения с ними как в дистанционно пилотируемом, так и в автономном режимах полета;

учреждения государственных и международных органов по лицензированию деятельности государственного и частного сектора в области использования БПЛА;

реализации жестких мер охраны и контроля БПЛА с целью недопущения их использования для террористических актов;

использования значительного количество БПЛА для обеспечения устойчивой передачи данных в условиях сложного рельефа или сложных метеоусловий;

создания эффективной системы подготовки обслуживающего персонала БПЛА и высокотехнологической инфраструктуры мест их базирования, переоснащения и ремонта.

Целью данной статьи является разработка концепции построения интегрированной системы послеаварийного мониторинга АЭС и поддержки принятия решений, которая, несмотря на повреждения при аварии, обеспечит высокую надежность обмена данными с измерительными (и при определенных условиях - управляющими) каналами для принятия обоснованных решений. Такая система может осуществлять мониторинг не только соответствующего оборудования, но и пространственный мониторинг (помещений и территорий).

Основные требования к системам послеаварийного мониторинга тяжелых аварий. На сегодняшний день основными системами, используемыми в Украине для послеаварийного мониторинга тяжелых аварий, являются система послеаварийного мониторинга (ПАМС) [9] и автоматизированная система контроля радиационной обстановки (ACKPO) [10].

П А М С предназначена для контроля параметров безопасности и состояния систем реакторной установки во время проектных и запроектных (в том числе связанных с тяжелым повреждением топлива) аварий на АЭС в условиях максимального расчетного землетрясения и полного обесточивания энергоблока.

Основные требования к системе ПАМС:

осуществление информационной поддержки персонала и экспертов по безопасности во время управления авариями, ликвидации их последствий и возвращения реакторной установки в контролируемое состояние, а также в процессе последующего анализа причин возникновения и путей протекания проектных и запроектных (в том числе тяжелых) аварий;

выполнение функций архивирования, отображения и регистрации данных о возникновении исходных событий, аварийных ситуаций и аварий, о действиях систем безопасности и персонала, направленных на обеспечение безопасности, а также данных о контролируемых параметрах, характеризующих состояние реактора, технологического оборудования, физических барьеров и других конструкций, систем и элементов, важных для безопасности;

охват диапазонами измерений контролируемых ПАМС параметров тех значений, при которых могут быть нарушены целостность тепловыделяющих элементов, а также границы теплоносителя первого контура герметичного ограждения;

выполнение основных функций при любых учитываемых проектом исходных событиях, нарушениях пределов и условий нормальной и безопасной эксплуатации, в аварийных ситуациях, во время развития аварий и в послеаварийных режимах;

сохранение данных в архиве, в том числе их неповрежденность в условиях запроектных аварий.

А С К Р О является системой, обеспечивающей контроль за радиационной обстановкой на территории АЭС, санитарно-защитной зоны, зоны наблюдения наряду с другими системами радиационного контроля, обеспечивающими контроль целостности последовательных барьеров на пути выхода радиоактивных веществ в окружающую среду для реализации технических и организационных мер по защите и предотвращению повреждения самих барьеров.

Основные требования к системе АСКРО:

непрерывность контроля параметров, характеризующих радиационную обстановку на промплощадке АЭС, в санитарно-защитной зоне, в зоне наблюдения при нормальной эксплуатации АЭС, при запроектных авариях, а также радиационную обстановку при прекращении эксплуатации АЭС;

прогнозирование радиационной обстановки на промплощадке АЭС, в санитарно-защитной зоне и зоне наблюдения во всех режимах работы АЭС, включая запроектные аварии;

обеспечение связи и обмена информацией со смежными системами.

Концепция построения систем мониторинга с использованием БПЛА. Во время аварии часть проводных линий связи может отказать. Вероятность полной потери связи (со всеми измерительными каналами), даже при серьезной аварии, невысока. Однако при повреждении части линий связи соответствующие потоки информации, в лучшем случае, должны принять на себя коммуникации, которые не повреждены, а значит, возрастают частота коллизий и вероятность локальной перегрузки сетей. Это создает дефицит информации именно тогда, когда она наиболее необходима. Для выхода из этой ситуации можно использовать дополнительные беспроводные каналы связи, которые обеспечат нужную пропускную способность и восстановят связь с теми измерительными модулями, ведущие каналы которых повреждены. Для обеспечения передачи информации в качестве ретрансляторов, а также для ее измерения при определенных условиях используются БПЛА.

Принципы построения систем мониторинга с использованием БПЛА. Основными свойствами системы послеаварийного мониторинга АЭС $[6-8,11]$ должны быть информативность, надежность и достоверность.

Обеспечение указанных свойств в послеаварийных условиях при использовании беспроводной связи является непростой задачей, поскольку измерительные 
и управляющие модули могут находиться в помещениях или под завалами, что значительно ослабляет уровень сигнала. При этом на АЭС уровень электромагнитных помех в послеаварийных условиях может возрастать. Таким образом, обеспечение непосредственной беспроводной связи между измерительными и управляющими модулями и центром управления требует значительной мощности передатчиков. Но вероятность повреждения при аварии сети питания измерительных и управляющих модулей тоже очень высока, поэтому модули и их узлы беспроводной связи будут питаться от аварийного аккумулятора. В таком случае мощные передатчики значительно снизят живучесть системы послеаварийного мониторинга за счет ограничения времени работы без восстановления заряда аккумулятора.

Обеспечить бесперебойное длительное (рациональное с точки зрения энергопотребления) функционирование системы послеаварийного мониторинга на базе беспроводной сети в условиях высокого уровня помех (т. е. обеспечить информативность системы) можно только значительно (во много раз) уменьшив расстояние беспроводной связи и существенно повысив устойчивость такой связи к помехам. Первое требование можно выполнить, применив промежуточные ретрансляционные модули, которые должны быть подвижными для того, чтобы: 1) до аварии находиться на расстоянии, практически исключающем их повреждение при аварии; 2) достаточно быстро, в течение минут, занять нужное место в соответствии с характером аварии, уровнем помех и расстоянием до обслуживаемых измерительных и управляющих модулей; 3) передвигаться (менять место дислокации) в соответствии с изменением условий, указанных в п. 2).

Выполнить перечисленные требования легче, если перемещать ретрансляционные модули воздушным путем. Довольно широкое распространение в последнее время получили дроны [6-8]. Именно они на сегодня являются наиболее выгодной платформой, где могут быть размещены ретрансляционные модули. Такое решение обеспечивает максимальную возможность доступа на нужную позицию и высокую скорость перемещения. Сеть ретрансляторов на основе дронов, которые базируются на значительном расстоянии от АЭС и вылетают на позиции после возникновения аварии, может обеспечить нужный поток данных за минуты после возникновения аварии.

Для повышения устойчивости беспроводной связи к помехам нужно применять коды с максимальными возможностями обнаружения и коррекции ошибок. Однако в условиях высокого уровня помех необходимо обеспечить помехоустойчивость не только данных и команд, а и высокую помехоустойчивость самого обмена. Известные протоколы рассчитаны на обычный уровень помех. Они не будут нормально функционировать при аварии на АЭС. Следует разработать протокол обмена данными, который будет иметь допустимый уровень помех, соответствующий наиболее жестким послеаварийным условиям на АЭС. Высокая помехоустойчивость требует использования избыточных кодов, поэтому для уменьшения трафика нужно перед кодированием максимально сжать данные от датчиков.

Для обеспечения достаточной пропускной способности каналов связи необходимо предусмотреть возможность распараллеливания потоков данных, т. е. распределения обслуживаемых измерительных и управляющих модулей между отдельными ретрансляторами. Чтобы поддерживать информативность системы послеаварийного мониторинга на достаточном уровне (а следовательно, и ее живучесть), требуется динамическая адаптация ретрансляторов и программно-техническая реконфигурация структуры при изменении условий функционирования системы. Отдельные ретрансляторы в послеаварийных условиях могут быть повреждены или возвращены на место базирования для восстановления заряда аккумуляторов. Их трафик должны взять на себя другие ретрансляторы.

Надежность системы послеаварийного мониторинга обеспечивается, во-первых, резервным аккумуляторным питанием измерительных и управляющих каналов. Для продления времени функционирования системы заряд аккумуляторов нужно максимально экономить, используя не только энергосберегающее аппаратное обеспечение, но и снижая (насколько возможно) мощность передатчиков беспроводной связи за счет уменьшения расстояния между ретрансляторами и обслуживаемыми ими измерительными и управляющими модулями. Упомянутая проблема минимизации энергопотребления может быть решена либо оптимизацией распределения обслуживаемых измерительных и управляющих модулей между отдельными ретрансляторами, либо использованием динамической оценки уровня ошибок при обмене данными и адаптацией мощности передатчиков.

Во-вторых, высокая надежность системы послеаварийного мониторинга обеспечивается свойствами сети ретрансляторов. Ретрансляторы, доставляемые на место аварии после ее возникновения (они не могут быть повреждены при аварии), должны динамически перераспределять потоки данных, оптимизировать свое положение относительно обслуживаемых измерительных и управляющих модулей, а также учитывать рельеф местности и наличие механических препятствий. Дроны-ретрансляторы должны восстанавливать заряд аккумуляторов при временном возвращении на место базирования, а поврежденные дроны - заменяться другими из предусмотренного резерва.

Высокая достоверность потока данных обеспечивается самотестированием каналов связи ретрансляторов, причем без частого выполнения указанной процедуры. Использование помехоустойчивых кодов с коррекцией ошибок позволяет осуществлять текущий контроль каналов связи по уровню ошибок. Уровень ошибок одновременно характеризует уровень помех и формирует информацию для подсистемы оптимального размещения дронов. При условии высокого уровня ошибок, но приемлемого для данной системы помехоустойчивого кодирования, возможен выбор места и мощности передатчика.

Измерительные и управляющие модули могут быть повреждены как во время аварии, так и после нее (например, в результате проникающей радиации). Поэтому достоверности данных можно достичь, в частности, оснащением измерительных и управляющих модулей автономными подсистемами метрологического обеспечения. Такие подсистемы должны быть устойчивыми к погрешностям их компонентов, которые могут резко возрастать в послеаварийных условиях (например, за счет действия проникающей радиации).

Организация функционирования флота дронов для системы послеаварийного мониторинга основывается на следующих принципах:

1. Флот дронов постоянно базируется на значительном расстоянии от АЭС и разворачивается после аварии. 
2. Флот включает дроны-ретрансляторы, дроны-наблюдатели (оснащены телекамерой) и дроны-датчики. Дроны должны иметь возможность изменять назначение благодаря переоснащению на месте базирования.

3. Дроны-ретрансляторы взаимодействуют согласно принципу «один ведущий». Такой принцип обеспечивает максимальную надежность функционирования системы беспроводной связи (возникает минимум коллизий). При возможном повреждении «ведущего» его функции принимает другой дрон-ретранслятор, например тот, который имеет наименьшее время наработки среди задействованных на месте аварии.

4. «Ведущий» дрон-ретранслятор определяет зону размещения каждого другого дрона-ретранслятора и порядок взаимодействия с измерительными модулями.

5. Каждый дрон-ретранслятор самостоятельно выбирает место с минимальным уровнем помех, необходимую мощность ретрансляции для измерительных модулей по уровню ошибок в передаваемых данных. Кроме того, по решению кризисного центра может осуществить посадку для проведения измерений.

6. Дроны-наблюдатели дают возможность постоянного визуального мониторинга места аварии для оценки действий дрона другого назначения, выбора безопасных мест приземления дронов-ретрансляторов, оценки траектории движения дронов-датчиков и места их нахождения.

Структура системы. Структурная схема постоянно действующей проводной сети измерительных и управляющих модулей и ее беспроводного расширения представлена на рис. 1.
Датчики и исполнительные механизмы от 1 до $n$ подключены к $m$ традиционным измерительным и управляющим модулям. Каждый из них включает многоканальный аналого-цифровой преобразователь (АЦП) или цифроаналоговый преобразователь (ЦАП), микроконтроллер традиционной обработки данных и адаптер проводного интерфейса.

Через этот интерфейс измерительные и управляющие модули взаимодействуют с центром управления и принятия решений - получают команды и пересылают результаты измерения. Для работы измерительных и управляющих модулей в составе системы послеаварийного мониторинга каждый из них оснащен дополнительным микроконтроллером, который принимает данные с беспроводной сети или готовит данные для передачи через нее (беспроводной микроконтроллер).

Микроконтроллер получает результаты измерения (от микроконтроллера измерительного модуля или от адаптера ведущего интерфейса), сжимает их, кодирует и передает через беспроводный интерфейс. Измерительные и управляющие модули при отсутствии напряжения сети их питания питаются от автономного батарейного блока (на схеме не показан).

В режиме нормальной эксплуатации обмен данными и командами происходит через проводную сеть. При ее повреждении во время аварии создается беспроводная сеть на базе дронов. По командам «ведущего» они размещаются в воздухе таким образом, чтобы: охватить все измерительные модули, оснащенные беспроводной связью; распределить потоки данных через дроны по возможности

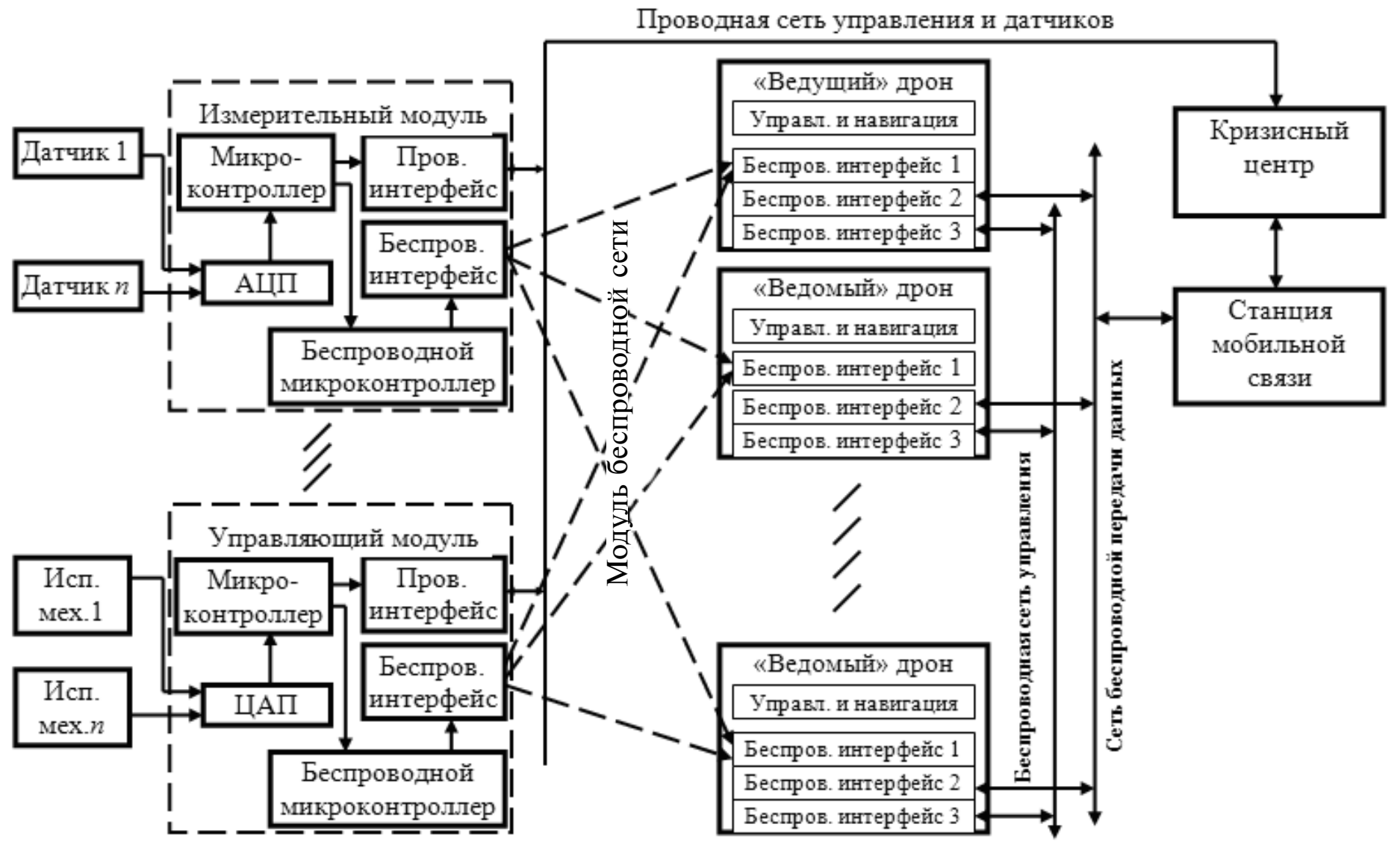

Рис. 1. Структурная схема проводной сети измерительных и управляющих модулей и ее беспроводного расширения 
равномерно, обеспечить максимальную достоверность передачи данных от датчиков и команд управления; не сталкиваться с препятствиями; не создавать помех друг другу.

При автономном питании измерительных и управляющих модулей только от резервных аккумуляторов очень важно минимизировать их энергопотребление, используя все возможности, в том числе ограничения мощности передатчика беспроводного интерфейса. Дроны в этом случае следует разместить в соответствующих зонах, приближенных к источникам сигнала. Энергосбережение может обеспечиваться за счет управления уровнем ошибок при обмене сообщениями. Если уровень ошибок приемлемый для выбранного метода кодирования, можно попробовать уменьшить мощность передатчиков беспроводного интерфейса как в составе измерительных модулей, так и в составе дронов.

Кроме того, дроны должны быть оснащены качественной системой навигации. Такая система должна обеспечивать:

1) возможность использования существующей системы глобальной навигации (GPS). Отметим, что в послеаварийных условиях на АЭС резко повышается уровень помех. В то же время сигнал от спутников GPS довольно слабый. Вероятность того, что он может оказаться недостаточным для надежной навигации, очень высока;

2) возможность использования локальной системы маяков, аналогично GPS. Расстояние до локальных маяков (источников индивидуальных сигналов, местоположение которых привязано к локальной карте местности) может быть во много тысяч раз меньше, и обеспечение необходимого уровня сигнала, даже при питании от аккумуляторов, не будет проблемой;

3) защиту от столкновений с местными препятствиями при помощи ультразвуковой локации. При этом ультразвуковые зондирующие импульсы должны иметь уникальное индивидуальное кодирование, иначе импульсы других дронов будут приниматься за свои, делая систему защиты от столкновений с препятствиями неработоспособной.

Необходимо отметить, что система ультразвуковой локации слишком неточная и в динамических условиях работает весьма медленно. Она не сможет воспрепятствовать столкновениям дронов между собой. Оценка вариантов защиты от таких столкновений показала, что лучшим вариантом будет учет позиций всех дронов одним «ведущим» и выдача им команд о позиции и векторах движения каждого (принцип Master-Slave). Однако при повреждении «ведущего» (Master) система становится неработоспособной. Поэтому необходимо предусмотреть возможность, при отсутствии команд Master, принятия на себя его функции каждым (или определенным) дроном. Во избежание коллизий функции Master целесообразно принимать по иерархическому принципу.

Отметим также, что сбор данных датчиков и управления исполнительными механизмами (обмен в сети измерительных и управляющих модулей), ретрансляция этих данных (обмен сообщениями с центром принятия решений и управления) и управления дронами (по командам «ведущего») - разные задачи, которые имеют мало общего. Разве что при выполнении задачи обмена в сети измерительных и управляющих модулей может быть определен уровень ошибок, и эта информация должна учитываться при выборе места дислокации дрона. Поэтому для повышения надежности функционирования системы послеаварийного мониторинга целесообразно разделить решения указанных задач на аппаратном уровне. Их должны выполнять различные микроконтроллеры, оснащенные собственными периферийными устройствами. При этом целесообразно формировать три независимые беспроводные сети обмена данными (измерительных и управляющих модулей, передаваемых данных и управления дронами), которые не будут конфликтовать между собой, создавать очереди и т. п.

\section{Выводы}

Предлагаемая концепция построения систем послеаварийного мониторинга АЭС на базе дронов обеспечивает выполнение требований к информативности, надежности и достоверности. Такой подход позволяет избежать неприемлемых повреждений и критического отказа системы послеаварийного мониторинга во время аварии, обеспечивает минимальное время развертывания системы, достаточную пропускную способность каналов связи, возможности ее наращивания и гибкого использования для решения других задач (доставки датчиков и другого оборудования в необходимое место), восстановление функционирования при повреждении компонентов.

\section{Список использованной литературы}

1. Morichi, M., Toubon, H., Bronson, F., Venkataraman, R., Beaujoin, J., Dubart, P. (2013), "Nuclear Measurement Technologies \& Solutions Implemented During Nuclear Accident at Fukushima", Advancements in Nuclear Instrumentation Measurement Methods and their Applications (ANIMMA), 2013 3rd International Conference, pp. $1-8$.

2. Vetter, K.,Chivers, D. H., Aucott, T., Bates, C., Coffer, A., Curtis, J., Hogan, D., Iyengar, A, Looker, Q., Miller, J., Negut, V., Plimley, B., Satterlee, N., Supic, L., Yee, B. (2011) "Measurements of Fukushima fallout by the Berkeley Radiological Air and Water Monitoring project", Nuclear Science Symposium and Medical Imaging Conference (NSS/MIC), pp. 18-24.

3. Torii, T., Sanada, Y., "Radiation measurement by unmanned aircraft after Fukushima Daiichi nuclear power plant accident", Remotely Piloted Aircraft Systems Symposium ICAO Headquarters, Montrŭal, Canada 23 - 25 March 2015, URL: http://www.icao. int/Meetings/RPAS/RPASSymposiumPresentation/Day\%201\%20 Session\%202\%20Massaki\%20Nakadate.pdf.

4. Cai, C., Carter, B., Srivastava, M., Tsung, J., Vahedi-Faridi, J., and Caroline, W. (2016) "Designing a Radiation Sensing UAV System", 2016 IEEE Systems and Information Engineering Design Conference (SIEDS '16), pp. 165-169.

5. "A new UAV for background radiation monitoring is developed for Russian meteorology and ecological monitoring corporation Rosgidromet”, URL: http://unmanned.ru/en/news/2014-06-02.htm.

6. Саченко А. О., Кочан В. В., Харченко В. С., Яцків В. В., Чернишов М. А., Биковий П. Є., Рощупкін О. Ю., Коваль В. С. Концепція побудови мобільних систем пост-аварійного моніторингу АЕC 3 використанням флоту квадрокоптерів. Радіоелектронні і комп’ютерні системи. 2016. № 5 (79). С. 207214.

7. Kharchenko, V., Sachenko, A., Kochan, V., Fesenko, H. (2016), "Reliability and Survivability Models of Integrated Drone-Based Systems for Post Emergency Monitoring of NPPs", Proceeding of The International Conference on Information and Digital Technologies 2016, IDT 2016, July 5-7, 2016, Rzeszow, Poland, pp. 127-132 (IEEE Catalog Number CFP16CDT-USB).

8. Sachenko, A., Kochan, V., Kharchenko, V., Roth, H., Yatskiv, V., Chernyshov, M., Bykovyy, P., Roshchupkin, O., Koval, V., Fesenko, H. (2016), "Mobile Post-Emergency Monitoring System for Nuclear Power Plants", ICT in Education, Research and Industrial 
Applications : Integration, Harmonization and Knowledge Transfer, Proceedings of the 12th International Conference, ICTERI 2016, June 21-24, 2016, Kyiv, Ukraine, pp. 384-398.

9. Инюшев В. В., Трубчанинов С. А., Ястребенецкий М. А. Регулирование безопасности применительно к системам аварийного и послеаварийного мониторинга АЭС. Ядерна та радіаційна безпека. 2013. № 3 (59). С. 48-53.

10. Вестрон. АСКРО ЗАЭС. Техническое задание. ТЗ ВН. 702.410.34. Харьков, 2011. 124 c.

11. Hiromoto, R. E., Sachenko, A., Kochan, V., Koval, V., Turchenko, V., Roshchupkin, O., Yatskiv, O., Kovalok, K. Mobile Ad Hoc Wireless Network for Pre- and Post-Emergency Situations in Nuclear Power Plant, The 2nd IEEE International Symposium on Wireless Systems within the Conferences on Intelligent Data Acquisition and Advanced Computing Systems, Offenburg, Germany, 2014, pp. 92-96.

\section{References}

1. Morichi, M., Toubon, H., Bronson, F., Venkataraman, R., Beaujoin, J., Dubart, P. (2013), "Nuclear Measurement Technologies \& Solutions Implemented During Nuclear Accident at Fukushima", Advancements in Nuclear Instrumentation Measurement Methods and their Applications (ANIMMA), 3rd International Conference, pp. 1-8.

2. Vetter, K.,Chivers, D. H., Aucott, T., Bates, C., Coffer, A., Curtis, J., Hogan, D., Iyengar, A, Looker, Q., Miller, J., Negut, V., Plimley, B., Satterlee, N., Supic, L., Yee, B. (2011) "Measurements of Fukushima Fallout by the Berkeley Radiological Air and Water Monitoring project", Nuclear Science Symposium and Medical Imaging Conference (NSS/MIC), pp. 18-24.

3. Torii, T., Sanada, Y. (2015), "Radiation Measurement by Unmanned Aircraft after Fukushima Daiichi Nuclear Power Plant Accident", Remotely Piloted Aircraft Systems Symposium ICAO Headquarters, Montrŭal, Canada, available at: http://www.icao. int/Meetings/RPAS/RPASSymposiumPresentation/Day\%201\%20 Session\%202\%20Massaki\%20Nakadate.pdf

4. Cai, C., Carter, B., Srivastava, M., Tsung, J., Vahedi-Faridi, J., and Caroline, W. (2016), "Designing a Radiation Sensing UAV System", IEEE Systems and Information Engineering Design Conference (SIEDS '16), pp. 165-169.

5. "A New UAV for Background Radiation Monitoring is Developed for Russian Meteorology and Ecological Monitoring Corporation Rosgidromet”, available at: http://unmanned.ru/en/news/2014-06-02. htm

6. Sachenko, A. O., Kochan, V. V., Kharchenko, V. S., Yatskiv, V. V., Chernyshov, M. A., Bykovyi, P. Ye., Roshchiupkin, O. Yu., Koval, V. S. (2016), "Concept of Constructing Mobile Systems of Post-Accident NPP Monitoring Using Quadcopters Fleet" [Kontseptsiia pobudovy mobilnykh system post-avariinoho monitorynhu AES z vykorystanniam flout kvadrokopteriv], Radioelectronic and Computer Systems, No. 5, pp. 207-214. (Ukr)
7. Kharchenko, V., Sachenko, A., Kochan, V., Fesenko, H. (2016), "Reliability and Survivability Models of Integrated Drone-Based Systems for Post Emergency Monitoring of NPPs", Proceeding of the International Conference on Information and Digital Technologies, IDT 2016, Rzeszow, Poland, pp. 127-132 (IEEE Catalog Number CFP16CDT-USB).

8. Sachenko, A., Kochan, V., Kharchenko, V., Roth, H., Yatskiv, V., Chernyshov, M., Bykovyy, P., Roshchupkin, O., Koval, V., Fesenko, H. (2016), "Mobile Post-Emergency Monitoring System for Nuclear Power Plants", ICT in Education, Research and Industrial Applications: Integration, Harmonization and Knowledge Transfer, Proceedings of the 12th International Conference, ICTERI 2016, Kyiv, Ukraine, pp. 384398.

9. Iniushev, V. V., Trubchaninov, S. A., Yastrebenetsky, M. A. (2013), "Safety Regulation of Emergency and Post-Accident NPP Monitoring Systems" [Regulirovaniie bezopasnosti primenitelno k sistemam avariinogo i posleavariinogo monitoringa AES], Nuclear and Radiation Safety, No. 3 (59), pp. 48-53 (Rus)

10. Westron, ZNPP Automated System for Radiation Situation Monitoring, Terms of Reference, TZ - VN, 702.410.34 [Westron. ASKRO ZAES. Tekhnicheskoiie zadaniie. TZ - VN. 702.410.34], Kharkiv, 2011, 124 p. (Rus)

11. Hiromoto, R. E., Sachenko, A., Kochan, V., Koval, V., Turchenko, V., Roshchupkin, O., Yatskiv, O., Kovalok, K. (2014), "Mobile Ad Hoc Wireless Network for Pre- and Post-Emergency Situations in Nuclear Power Plant", 2nd IEEE International Symposium on Wireless Systems within the Conferences on Intelligent Data Acquisition and Advanced Computing Systems, Offenburg, Germany, pp. 92-96. 